\title{
Neuro-Sweet disease with positive modified acid-fast staining of the cerebrospinal fluid: A case report
}

\author{
JUAN-FANG LIU ${ }^{1,2^{*}}$, YUAN LI $^{3 *}, \mathrm{KAI} \mathrm{LI}^{4}$, XIAO ZHANG ${ }^{1}$, YI-NING YANG ${ }^{1}$, GANG ZHAO $^{1}$ and ZHI-RONG LIU ${ }^{1}$ \\ ${ }^{1}$ Department of Neurology, Xijing Hospital, Fourth Military Medical University; ${ }^{2}$ Department of Clinical Aerospace Medicine, \\ Key Laboratory of Aerospace Medicine of Ministry of Education, Fourth Military Medical University, Xi'an, Shaanxi 710032; \\ ${ }^{3}$ Department of Clinical Medicine, Second Clinical Medical College, Southern Medical University, Guangzhou, \\ Guangdong 510515; ${ }^{4}$ Department of Dermatology, Xijing Hospital, Fourth Military Medical University, \\ Xi'an, Shaanxi 710032, P.R. China
}

Received November 21, 2014; Accepted December 21, 2015

DOI: $10.3892 /$ etm.2016.3052

\begin{abstract}
Neuro-Sweet disease (NSD) is Sweet disease with central nervous system (CNS) involvement. To the best of our knowledge, the present case report is the first to describe NSD complicated by endogenous infection with Mycobacterium tuberculosis. The present case report describes a male patient who developed NSD-induced meningitis, which initially manifested as a fever, headache and neck stiffness. Painful erythematous plaques subsequently developed on his face, neck and upper trunk. Brain magnetic resonance imaging was performed and the results were normal, whereas modified acid-fast stain analysis of the cerebrospinal fluid (CSF) provided a positive result. The patient was thus diagnosed with viral meningitis and tuberculosis. However, subsequent skin biopsy results demonstrated neutrophilic infiltration into the dermis without vasculitis, and subsequent human leukocyte antigen typing was positive for Cw1 and negative for B51 and the patient was diagnosed with NSD. Following treatment with corticosteroids, and antiviral and anti-tuberculotic agents, the clinical symptoms were reduced and the previously abnormal findings in the CSF examinations and associated laboratory data were improved. The present case indicates that the diagnosis of NSD is not easily achieved, and early skin biopsy is vital to ensure a fast and effective diagnosis. In addition to systemic corticosteroids, comprehensive treatment is also recommended for patients with NSD complicated by additional complex medical problems.
\end{abstract}

Correspondence to: Dr Zhi-Rong Liu, Department of Neurology, Xijing Hospital, Fourth Military Medical University, 127 West Changle Road, Xi'an, Shaanxi 710032, P.R. China

E-mail: liuzhir@fmmu.edu.cn

*Contributed equally

Key words: acute febrile neutrophilic dermatosis, human leukocyte antigen, meningitis, modified acid fast stain, neuro-Sweet disease

\section{Introduction}

Sweet disease, which is also known as acute febrile neutrophilic dermatosis, is a multisystemic inflammatory disease that was first described in 1964 (1). It is characterized by fever, peripheral neutrophil leukocytosis and tender erythematous skin lesions that heal without scarring. The skin lesions of Sweet disease are commonly asymmetrical and are predominantly located on the face, neck and upper extremities (1). Cutaneous manifestations of the disease may be preceded by a fever lasting from several days to weeks (2). Skin biopsies of cutaneous edematous erythematous plaques reveal deep dermal infiltration of mature neutrophils and the absence of vasculitis (3). Several cases of Sweet disease with CNS involvement have been reported (4-7) and neuro-Sweet disease (NSD) has been proposed as a novel concept (8). Although the aetiology of NSD remains elusive, it has previously been suggested that NSD is a distinct clinical entity that may account for various cases of idiopathic encephalitis or meningitis $(8,9)$. Systemic corticosteroid therapy is highly effective for the treatment of the majority of the neurologic symptoms detected in patients with NSD $(7,10,11)$. It may be useful to classify NSD as benign, however, there is a high frequency of symptom relapse $(8,9)$. In the present case report, a rare case of NSD with normal brain magnetic resonance imaging (MRI) and positive modified acid-fast staining of the cerebrospinal fluid (CSF). The patient's meningitis and skin lesions were successfully treated with systemic corticosteroids, and antiviral and anti-tuberculotic combination therapy.

\section{Case report}

In July 2013, a previously healthy 30-year-old Chinese man presented with headache, neck stiffness and fever at the First Affiliated Hospital of Xi'an Jiaotong University (Xi'an, China). Although these symptoms were alleviated following treatment with antibiotics, including $1 \mathrm{~g}$ compound polymyxin B ointment (Zhejiang Reachall Pharmaceutical Co., Ltd., Zhejiang, China) administered thrice daily and $3.75 \mathrm{~g}$ intravenous mezlocillin sodium and sulbactam sodium (Shanxi C\&Y Pharmaceutical Group Co., Ltd., Datong, China) administered 

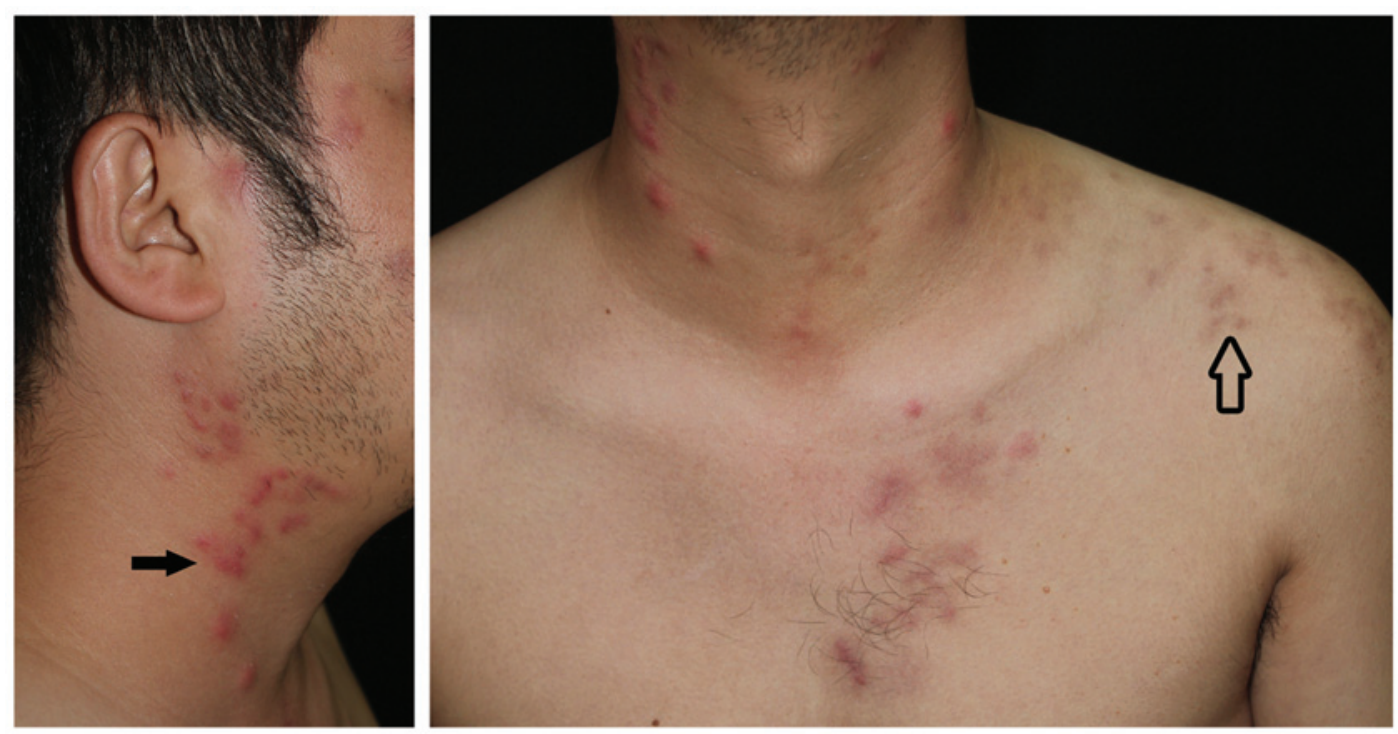

Figure 1. Cutaneous manifestations on the patient. Dull-red, erythematous plaques were present on the patient's face, neck and upper chest (solid arrow), and pigmentation from previous erythematous plaques was detected on his left shoulder (hollow arrow).

twice daily, several painful nodules subsequently developed on the patient's forehead. Two days later, the patient's headache and neck stiffness symptoms had worsened and intermittent fever $\left(38-39^{\circ} \mathrm{C}\right)$ persisted. Nodules subsequently developed on the patient's face, neck and chest; therefore, antiviral drugs, including $0.3 \mathrm{~g}$ ribavirin granules (Sichuan Baili Pharmaceutical Co., Ltd., Chengdu, China) twice daily and acyclovir ointment (q.s.; Fujian Pacific Pharmaceutical Co., Ltd., Jinjiang, China) every $4 \mathrm{~h}$, . were administered for a presumed herpes zoster infection; however, the symptoms did not improve.

The patient was then admitted to Xijing Hospital (Xi'an, China) on 22 July, 2013. Upon physical examination, raised tender erythematous plaques were detected on the face, neck and upper trunk of the patient. Pigmentation on the left shoulder was also observed (Fig. 1).

Routine laboratory tests revealed the patient's white blood cell (WBC) count was $10.36 \times 10^{9} / 1$ (normal range, $3.5-9.5 \times 10^{9} / 1$ ) with $82.4 \%$ neutrophils, the erythrocyte sedimentation rate (ESR) was $99 \mathrm{~mm} / \mathrm{h}$ (normal range, $0-20 \mathrm{~mm} / \mathrm{h}$ ), and the serum C-reactive protein (CRP) level was $132 \mathrm{mg} / \mathrm{l}$ (normal range, $0-5 \mathrm{mg} / \mathrm{l})$. Serological tests demonstrated that the patient was positive for antibodies to Epstein-Barr virus; whereas tests for antibodies to hepatitis B and C viruses, human immunodeficiency virus and Treponema pallidum were negative. The lipopolysaccharide (LPS) level was $289 \mathrm{pg} / \mathrm{ml}$ (normal range, $0-10 \mathrm{pg} / \mathrm{ml}$ ) and the $1,3-\beta$-D-glucan level was within the normal range. Blood culture for bacteria and T-spot tuberculosis tests were negative. Extensive autoimmune screening revealed that the patient was positive for antinuclear antibodies, however he did not present with any of the typical symptoms of either systemic lupus erythematosus, mixed connective tissue disease, rheumatoid arthritis or dry syndrome; therefore this result was considered insignificant. Tumor marker tests revealed no evidence of malignancy.

A subsequent lumbar puncture demonstrated a pressure of $>300 \mathrm{mmH}_{2} \mathrm{O}$, and cytological analysis indicated that the CSF contained 80 leukocytes $/ \mathrm{mm}^{3}$ (83\% lymphocytes;

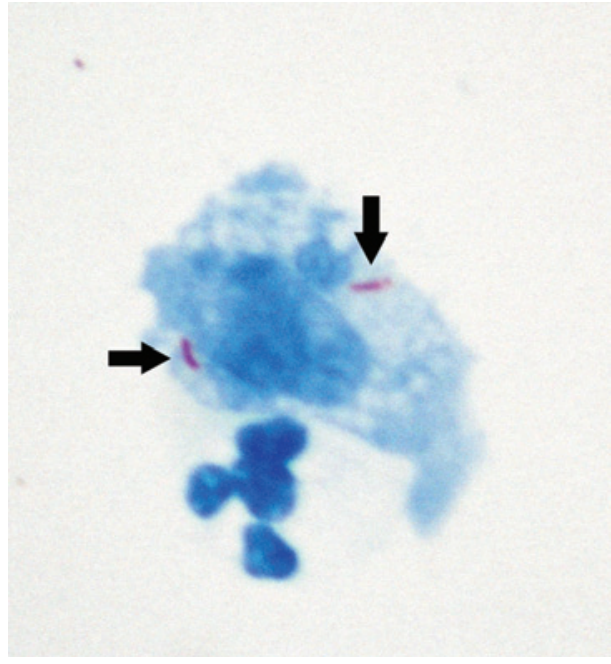

Figure 2. Modified acid fast staining of the cerebral spinal fluid sample demonstrated two acid-fast bacilli in a macrophage, as indicated by the arrows. (magnification, x100).

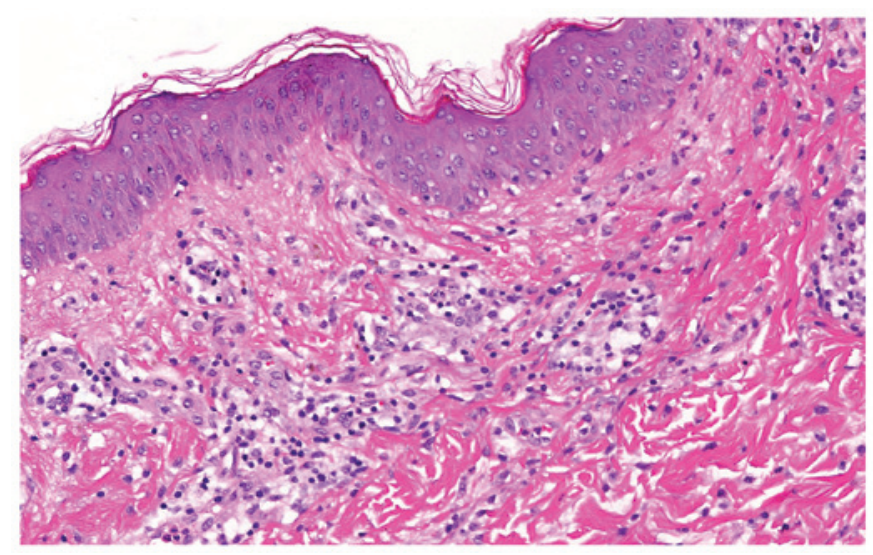

Figure 3. Histological photomicrograph. Dense neutrophil infiltration was detected in the dermis with no evidence of vasculitis in a biopsy specimen from one of the plaques, as detected by hematoxylin and eosin staining (magnification, $\mathrm{x} 20$ ). 
8.5\% mononuclear cells; $6 \%$ neutrophilic cells; and $2.5 \%$ plasma cells). These results were outside of the normal range and were outside of the normal range, suggesting inflammation in the patient's central nervous system. Immunoglobulin (Ig) $\mathrm{M}$ levels were raised at $2.69 \mathrm{mg} / \mathrm{l}$ (normal range, 0-1.0 mg/l), which indicated possible acute viral meningitis. Protein, glucose and chloride levels were normal. A modified acid-fast stain test was performed on the CSF sample according to the staining methods described by Feng et al (12), which gave a positive result (Fig. 2). Alcian blue staining demonstrated that the CSF was negative for Cryptococcus spp. Tests for antibodies against common viruses were negative, and subsequent brain MRI, electroencephalogram and X-ray examination of the chest did not detect any abnormalities.

Since these results initially suggested the patient was suffering from viral meningitis, intravenous ganciclovir (750 mg/day for 15 days) and dexamethasone $(20 \mathrm{mg} /$ day for 5 days, then $10 \mathrm{mg} /$ day for 5 days) was administered, followed by oral prednisone acetate tablet $(40 \mathrm{mg} /$ day, gradually tapered by $10 \mathrm{mg}$ weekly). On 25 July 2013, a biopsy was taken from one of the erythematous plaques on the left side of the patient's neck. The tissue sample was paraffin-embedded and serial $3 \mu \mathrm{m}$-thick sections were cut and subsequently stained with hematoxylin and eosin. Furthermore, the positive CSF modified acid-fast stain result indicated infection with Mycobacterium tuberculosis; therefore the patient received anti-tuberculotic therapy whilst the results of the skin biopsy were awaited. Anti-tuberculotic therapy included $0.45 \mathrm{~g}$ rifampicin capsules (q.d.), $0.5 \mathrm{~g}$ pyrazinamide tablets (both Chengdu Jinhua Pharmaceutical Co., Ltd., Chengdu, China) thrice daily, $0.6 \mathrm{~g}$ isoniazid (q.d.; Tianjin Kingyork Group Co., Ltd., Tianjin, China) via an intravenous drip, ganciclovir (Hidragon Pharmaceutical Co., Ltd., Shanghai, China), dexamethasone (Cisen Pharmaceutical Co., Ltd., Jining, China) and prednisone acetate (Tianyao Pharmaceutical Co., Ltd., Tianjin, China).

Following 3 days of treatment, the fever was alleviated, and a second lumbar puncture indicated a pressure of $225 \mathrm{mmH}_{2} \mathrm{O}$, and CSF analysis detected 27 leukocytes $/ \mathrm{mm}^{3}$ (96.5\% lymphocytes; $3.5 \%$ mononuclear cells; and $0 \%$ neutrophilic cells and plasma cells). The IgM level was low at $1.22 \mathrm{mg} / \mathrm{l}$, and the CSF modified acid-fast stain was negative. The WBC count was $8.90 \times 10^{9} / 1$ (70.2\% neutrophils; $26.5 \%$ lymphocytes; $3.1 \%$ monocytes; and $0.2 \%$ eosinophils), LPS was $<5.00 \mathrm{pg} / \mathrm{ml}$, and the ESR and CRP levels had decreased to $40 \mathrm{~mm} / \mathrm{h}$ and $0.92 \mathrm{mg} / \mathrm{l}$, respectively. These clinical findings indicated that treatment was effective and the patient was recovering well.

Subsequently, the skin biopsy demonstrated massive infiltration of neutrophils into the dermis and dermal edema without vasculitis (Fig. 3). These findings were consistent with Sweet disease. Furthermore, human leukocyte antigen (HLA) typing indicated the patient was positive for $\mathrm{Cw} 1$ and negative for B51. Cw1 and B51 HLA typing is often used for differential diagnosis of NSD (Positive Cw1 and negative B51) from neuro-Behçet disease (NBD) (Positive B51) (9). The differences between NSD and NBD are as follows: 1) Both sexes are evenly affected by NSD, whereas men are affected $\sim 3.4$ times as frequently as women with NBD; 2) individuals aged 30-70 are most commonly affected by NSD, whereas NBD preferentially affects those aged 20-40; 3) in NSD, any region of the CNS may be involved without site predilection, resulting in various neuro- logical symptoms, whereas the basal ganglia and brainstem are preferentially affected in NBD; 4) some patients with NSD demonstrate ocular signs, including episcleritis and conjunctivitis, whereas uveitis is common in NBD; and 5) there is a strong HLA-Cw1 and B54 association in NSD, whereas there is a high frequency of HLA-B51 in patients with NBD (9). As a result of the clinical features, CSF analysis and HLA typing, the patient was diagnosed with NSD, not NBD.

The patient was discharged following 17 days of hospitalization, at which point his headache and neck stiffness were alleviated and the erythematous plaques had receded without scarring. The patient was subsequently followed-up as an outpatient, and he remains well on a tapering dose of prednisone acetate tablets, oral aciclovir $(300 \mathrm{mg} / \mathrm{dose}, 3$ times/day for 6 days) and anti-tuberculotic drugs (for 3 months). At 15-month follow-up, the patient demonstrated no recurrence of symptoms or erythematous plaques. Written informed consent was obtained from the patient prior to publication.

\section{Discussion}

The patient presented with clinical features of viral meningitis and the cutaneous manifestations of classical Sweet disease. Although the brain MRI was normal, which has rarely been demonstrated in previous patients with NSD (4,9,13-16), the present patient fulfilled all the essential points of the diagnostic criteria for NSD, including: i) high fever of $38-40^{\circ} \mathrm{C}$; ii) painful and dull red erythematous plaques on the face, neck and upper part of the trunk; iii) neutrophilic infiltration of the dermis without uveitis; iv) positive HLA-Cw1 and negative HLA-B51; and v) good response to corticosteroid therapy (8). Combination therapy with corticosteroids and antiviral agents was initiated due to suspected viral meningitis, prior to the final diagnosis of NSD. In addition to corticosteroids and antiviral therapy, anti-tuberculotic agents were also administered as the CSF modified acid-fast stain was positive, which suggested that the patient was concurrently suffering from an endogenous infection of $M$. tuberculosis and, therefore, corticosteroid administration would have lead to the spread of M. tuberculosis. The patient responded well to these drugs. Given the rarity of NSD, it is understandable that the correct diagnosis was not immediately achieved. The present case reinforces the value of an early skin biopsy in order to confirm the diagnosis, avoid unnecessary therapy, allow the prompt initiation of the appropriate treatment and achieve successful therapeutic management.

\section{Acknowledgements}

The present study was supported by the National Natural Science Foundation of China (grant no. 81070950).

\section{References}

1. Sweet RD: An acute febrile neutrophilic dermatosis. Br J Dermatol 76: 349-356, 1964

2. Cohen PR: Sweet's syndrome - a comprehensive review of an acute febrile neutrophilic dermatosis. Orphanet J Rare Dis 2: 34, 2007.

3. Going JJ, Going SM, Myśkoẃ MW and Beveridge GW: Sweet's syndrome: Histological and immunohistochemical study of 15 cases. J Clin Pathol 40: 175-179, 1987. 
4. Hisanaga K, Hosokawa M, Sato N, Mochizuki H, Itoyama Y and Iwasaki Y: Neuro-sweet disease: Benign recurrent encephalitis with neutrophilic dermatosis. Arch Neurol 56: 1010-1013, 1999.

5. Noda K, Okuma Y, Fukae J, Fujishima K, Goto K, Sadamasa H, Yoshiike T and Mizuno Y: Sweet's syndrome associated with encephalitis. J Neurol Sci 188: 95-97, 2001.

6. Stenzel W, Frosch PJ and Schwarz M: Sweet's syndrome associated with acute benign encephalitis. A drug induced aetiology. J Neurol 250: 770-771, 2003.

7. Nobeyama Y and Kamide R: Sweet's syndrome with neurologic manifestation: Case report and literature review. Int J Dermatol 42: 438-443, 2003.

8. Hisanaga K, Iwasaki Y and Itoyama Y: Neuro-Sweet Disease Study Group: Neuro-Sweet disease: Clinical manifestations and criteria for diagnosis. Neurology 64: 1756-1761, 2005.

9. Hisanaga K: Neuro-neutrophilic disease: Neuro-Behcet disease and Neuro-Sweet disease. Intern Med 46: 153-154, 2007.

10. Dunn TR, Saperstein HW, Biederman A and Kaplan RP: Sweet syndrome in a neonate with aseptic meningitis. Pediatr Dermatol 9: 288-292, 1992.

11. Druschky A, von den Driesch P, Anders M, Claus D and Neundörfer B: Sweet's syndrome (acute febrile neutrophilic dermatosis) affecting the central nervous system. J Neurol 243 556-557, 1996.
12. Feng GD, Shi M, Ma L, Chen P, Wang BJ, Zhang M, Chang XL, Su XC, Yang YN, Fan XH, et al: Diagnostic accuracy of intracellular Mycobacterium tuberculosis detection for tuberculous meningitis. Am J Respir Crit Care Med 189: 475-481, 2014.

13. Kokubo Y, Kuzuhara S, Isoda K, Sato K, Kawada N and Narita Y: Neuro-Sweet disease: Report of the first autopsy case. J Neurol Neurosurg Psychiatry 78: 997-1000, 2007.

14. Niwa F, Tokuda T, Kimura M, Azuma Y, Mizuno T and Nakagawa M: Self-remitting and reversible parkinsonism associated with neuro-Sweet disease. Intern Med 49: 1201-1204, 2010.

15. Fukae J, Noda K, Fujishima K, Wada R, Yoshiike T, Hattori N and Okuma Y: Successful treatment of relapsing neuro-Sweet's disease with corticosteroid and dapsone combination therapy. Clin Neurol Neurosurg 109: 910-913, 2007.

16. Kimura A, Sakurai T, Koumura A, Suzuki Y, Tanaka Y, Hozumi I, Nakajima H, Ichiyama T and Inuzuka T: Longitudinal analysis of cytokines and chemokines in the cerebrospinal fluid of a patient with Neuro-Sweet disease presenting with recurrent encephalomeningitis. Intern Med 47: 135-141, 2008. 